\title{
Research on Construction and Practice of Public Elective MOOCs in Applied Undergraduate Colleges
}

\author{
Jie DING \\ Jilin Agricultural Science and Technology University, Jilin, China.
}

KEYWORD: MOOCs; Public elective; Construction and Practice

\begin{abstract}
MOOCs is the new teaching method, which has some merits to the traditional teaching in public elective courses, such as more professional, more flexible and suitable for students. In the paper, public elective MOOCs platform was constructed as well as its realization of the module is given with some text descriptions to relative public elective features. and it approves that MOOCs platform meets functional demands and performance demands by analyzing test results. In the end, it expounds the results achieved in the development and it proposes improvement direction for MOOCs's further development.
\end{abstract}

\section{GENERAL INSTRUCTIONS}

With the continuous development of technology, online education has been very popular, but new problems will arise, traditional online education is only for the actual transfer to the classroom a classroom network, did not highlight the benefits of online education where, and most College courses in small scale units, also limited the scope of the present college students, interaction between teachers and students are few. For problems now have a new solution, that is the lesson $\mathrm{Mu}$ internet. $\mathrm{Mu}$ Class platform has the following characteristics: Students only need to register an account on the network platform, you can log on to the internet, through a simple enrollment process will be able to learn a variety of courses provided by the platform, and the platform courses offered are diverse, each course is independent of each other, by the relevant teachers in maintenance. For learning the contents of the video are in accordance with short courses divided knowledge or with related programs in a few exercises, are not the kind of traditional classroom course about an hour. Because there are a lot of learning the number, the interactive learning to a higher demand for the exercises must implement real-time display scores, because with increasing student teachers for correcting exercises are unrealistic. As can be seen from the Feature class platform $\mathrm{Mu}, \mathrm{Mu}$ class is an open platform that can support a large number of students while online learning and can provide many courses conducted for a teaching platform management. According to the characteristics $\mathrm{Mu}$ class platform, the platform is the main problem is how to achieve a large number of students while online learning and courses on how to effectively manage for example, to maintain the relative independence between different courses, teachers should only be responsible for their own operations courses.

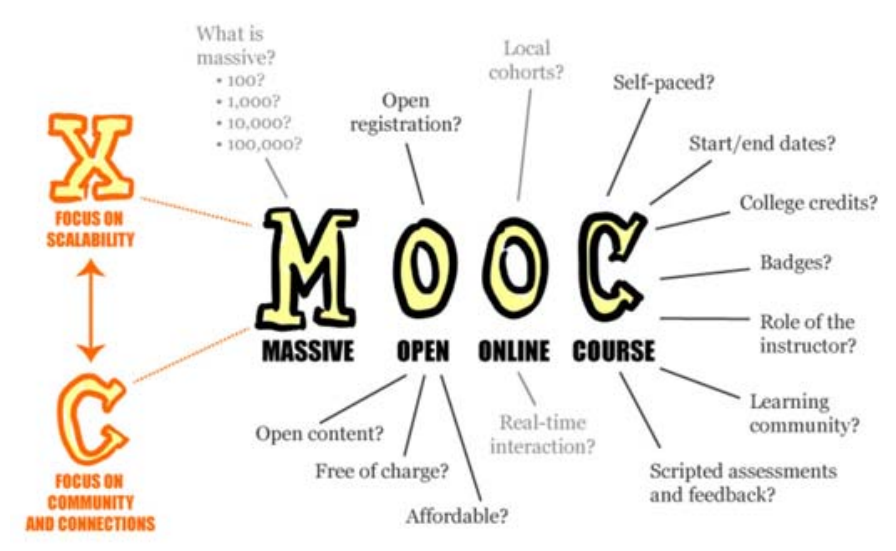

Figure 1. Historical Background Mu Course. 


\section{MU CLASS PLATFORM NEEDS ANALYSIS}

The overall objective of Mu class platform to provide users with an open register of teaching platform, capable of supporting a large number of students into the platform online learning, you can publish different courses, each course can be divided into sections of the curriculum structure, teachers can depending on the course structure for curriculum set the actual course content, such as video, exercises and so on. If you have questions about the program students can ask questions online to teachers, while teachers can respond to the problem. For information on courses courseware can be categorized according to the curriculum for unified management. Platform according to different user roles must meet the following three functions:

Administrators can platform for teachers and students to add, modify user information already exists, already present in the platform user delete operation. You can also maintain some static information and public information programs of courses, such as courses announcements, course introduction.

Teachers can publish courses through the platform, a teacher can publish many courses, but the teachers publish course is independent, that teachers can only control their own publishing programs can not operate on other teachers publish course. Courses for teachers has been published in accordance with section may set syllabus structure, can be added according to the structure of the curriculum subsections specific content. The course is not just a single section also includes video exercises and so on. Teachers can also publish courseware curriculum, according to the classification of the curriculum courseware for unified management. At the same time, teachers can also publish student problems reply to see students complete exercises resulting scores.

Students After logging platform, you can view all all courses teachers released, you can choose a course to learn according to your preference, mainly can operate for viewing the syllabus according to the course section, in accordance with subsection course video learning and practice to answer questions, and after the exercises can give the correct answer to complete the platform and to answer students' scores show that the answer obtained. Students can ask questions to teachers, according to the problem view your reply teachers.

\section{NON-FUNCTIONAL REQUIREMENTS ANALYSIS}

$\mathrm{Mu}$ class platform to meet the needs, in addition to achieve the functional requirements but also to achieve other than non-functional requirements.

Page response time due to platform design goal is to achieve a large number of students online learning it is necessary to ensure fast and accurate response pages, mainly in use within the company according to the existing experimental conditions and environment, the platform can support the requirements of the largest number of people online, to be able to ensure concurrent access platform enables the normal course release, watch online video and answer exercises. Since the platform in order to operate must first be logged in, so log in part also needs to carry more pressure, the response time should be less than seconds, the data within the course platform delivery time should be controlled within seconds, considering it comes to video processing will not be final including video page response time control in seconds, in the case that contains video page page response time control in seconds.

Security, security and confidentiality of the platform is an important non-functional requirements. User is not logged can not access any internet content, the platform will follow the line of authority into the role assigned to ensure that unauthorized behavior does not occur. For teachers publish course I can only operate in the case of increasing the number of courses, the phenomenon can not have access to each other's data between programs, in the case of increasing the number of students, the students have been chosen only for the course learning, there is no choice of courses can not learn, do exercises also should be able to score through a statistical summary of the information section of the course, the data can not appear the phenomenon of chaos.

Maintain and extend. Platform to have a certain scalability, because the platform is open for registration, the number of users will continue to improve, the course opened for this platform will continue to increase scalability is also required. For the expansion of the database platform database expansion should be done at the same time only need to modify some of the configuration file for the database, rather than to modify the source platform. 


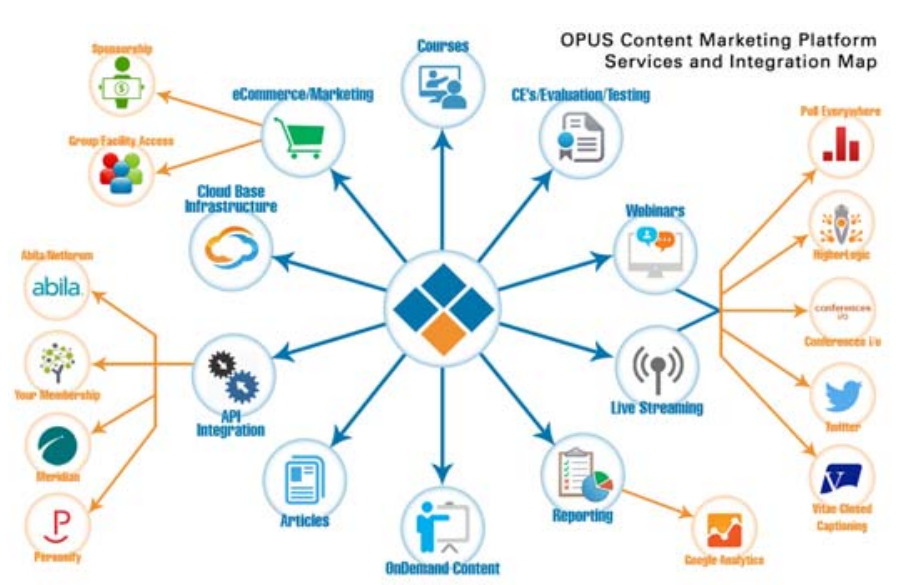

Figure 2. MOOCs platform flow chart.

JAX-WS Web Service specification is based on Java language, it is possible to develop a Web Service through service-oriented message (Message-Oriented) and process-oriented (RPC-oriented) in two ways. JAX-WS SOAP-based protocols to handle remote calls, functional interface developers only need to write code that announced good, JAX-WS will automatically generate messages on the SOAP protocol code it can be automatically generated client class by whimper tool file, packaged into a jar package introduces the project will be able to use the corresponding service. SOAP simple Object access protocol agreement is carried out simple communication protocol to pass data through the XML file, make different language applications of information when transferring data exchange. It consists of three parts: the pack data, process-oriented representation and encoding rules. These three parts are included in the XML file, the client application can parse XML file on the remote service call to the server release. The platform uses JAX-WS to achieve the flow and send the file to the Reds delete a file server function.

\section{MU CLASS DESIGN PLATFORM}

This chapter analyzes mainly based on demand platforms and the use case diagram analysis, design from a functional structure, module class diagram and a timing diagram the overall platform architecture, platform, and by the description of database diagram is given platform data model design, behind the platform coding pave the way.

Analysis of the structure of the platform, the platform is open for registration and therefore need the support of a large number of concurrent users to access the internet using Bis confirm architecture using Java EE enterprise application framework for the development of non-functional requirements through analysis, as well. Because open platform, so the amount of data platforms and the complexity of the data will be demanding, requires that the database should have distributed storage and data technologies such as cutting, decided to persistent data using SQL Server 2008 is complete, and SQL Server 2008 are also Great corresponding data support. Since the target platform to achieve large-scale open teaching platform Web server should be able to support load balancing technology for deploying Tomcat6 web servers, effectively reducing the overall cost of deployment also supports load balancing. Platform need to publish a large number of courses, but also need the support of a large number of students to watch, and determine its use Flex4 Reds play video server technology for processing video files.

For a complex platform, all resources should be orderly stored, curriculum resource management module main task is to curriculum related resources centralized management according to different courses. You need to associate curriculum resources and curriculum, in the course of growing conditions, to ensure the orderly classification of curriculum resources can not appear the phenomenon of cross-curriculum resources. Curriculum resources and resource management through the course id to bind store, so that all resources will have a unified curriculum identifies, when retrieving the resource as long as the id of the course will be able to filter out all the resources courses. Resource management module includes functions such as adding and deleting resources. Upload Resources page and video upload module using the same upload sub-module, resource management to achieve just added to the database, modify, and delete files. 
Design Resource Management Resource management module lasting design principle is based on the strategy pattern to design. For adding resources, delete, and query and other operations, you can define a common interface (HibernateDaoS upport, and then come together to achieve this interface through three different implementation classes implement add, delete, and query methods in each implementation class. then define a class announced different operations (ContentCRUT, define private variables in the class public interface, interface parameters passed in the constructor of the class. when using just call announced in class (ContentCRUT, and in the instance of the class when a different function to the constructor parameters can be achieved to add data, delete and modify functions such as resource management data persistence to member.

Analysis of the detailed design of the platform to determine platform architecture based on B / S browser and server architecture that is platform design patterns using the MVC pattern, respectively, using SSH2 framework of three-layer structure according to the needs of three lightweight $\mathrm{Mu}$ class platform frame. Platform video processing section on the use of video server technology design. According to the needs analysis data entity model yields the desired platform, based on the solid model and ER diagram for platform data model design. According to the functional structure of the platform division, the platform can be divided into user management module, management module syllabus, curriculum video management module, online test management module and curriculum resource management module. According to the different functional modules were designed in detail using the class diagram illustrates the internal structure of each module, a timing diagram illustrates the relationship between the various modules and internal calls sub-modules.

\section{MU ACHIEVE CLASS PLATFORM}

$\mathrm{Mu}$ class platform based on detailed design of complete coding platform to achieve, through the platform user management module is designed with sub-role management for large-scale student data management, design management module using the syllabus teachers and curriculum management ways data independence curriculum, curriculum management module uses video streaming technology for video playback pressure bearing, online test management module and curriculum resource management module to achieve results and statistics and remote resource management through Flex WebService service, specific details will be described by the flowchart Implementation process.

User access login.jsp page, select the correct identity of the drop-down list, and then type the login information, select the Login button to call login.jsp page checkl () function on the input data is empty for testing. After detection by calling LogService class login () method of user information stored in the database for comparison, if the teacher is logged in login information will be teachers (teachers entity "tea") to save the session object. If the login is administrator in the administrator's login information (the entity administrator "admin") to save the session object. login () method returns a value of type string of "yes" or "no". Upon completion also triggers login.jsp page callback match () function returns the database than to verify the results, if the return is "yes" will be called tiao loginSuccess.j sp page () function page jump , tiao () function will jump session object based on different types of entities. If the return is' 'no" in the pop-up window login.jsp page calls the method and return an error message pops up login.jsp page. After a successful login, if you are an administrator platform will jump to the index.jsp page admin directory. If it is teachers teachers platform will jump to the page atea directory.

For not through login and direct access to the internet page of the case will be used to intercept LogAOP class, LogAOP work steps: Spring reads the configuration file is information about AOP, and find the corresponding section implementation class (LogAOP according to the configuration information, configuration information the definition will be the starting point, the starting point would be the definition of a class or need to intercept method calls to be intercepted when the class or method, AOP will first perform a before () method prior to the implementation of the method currently executing method It has permission to judge. for there is no authority he will jump to the login page. 


\section{CONCLUSIONS}

In this paper, through the study of the existing online educational platform for the existing problems, combined domestic and international mainstream technical analysis. We completed the design and implementation of $\mathrm{Mu}$ class platform. Through the platform of each module analysis, the main achievements are:

First, the existing online educational platforms as well as the internal structure of the relevant literature analysis, and the actual situation of the Mu class platform should have the characteristics summarized and analyzed the main problem to be solved according to the characteristics of the platform the platform, and then to the platform for a detailed functional requirements analysis and non-functional requirements. Obtained according to the needs of the key technologies required for the development platform, the platform is open and there will be a large number of students studying at the same time, the platform formally adopted B / S architecture, the development model using the MVC design pattern, the layers in MVC member, use SSH2 architecture for understanding the lotus and high scalability. For internet data traffic the largest part of the video, the use of Flex technology and Reds video server technology. For the Reds video server upload and delete files using WebService technology.

For the primary problem to be solved by the platform, the platform can be divided functions include user management, course outline management, video management courses, online courses and test management resource management modules. SSH2 and use technology to achieve independence, course curriculum data published data only teachers can be accessed, and by the state curriculum and curriculum time to effectively control whether students can access the course; the use of Flex, Reds video server technology and WebService technology to solve course video Manager encountered remote upload video files, playback and delete other issues; the use of Flex technology solves the graphical component of student achievement statistics can not display graphical problems, so that the platform can follow the different sections of each segment of all scores student statistics and graphical display.

$\mathrm{Mu}$ class platform for functional and non-functional testing, and in the video portion of the conventional techniques are analyzed ActivaX comparison, proven Flex technology and Reds video server technology than traditional ActivaX technology from the stability and response time We have an advantage.

\section{REFERENCES}

Polhill, R.M. 1982. Crotalaria in Africa and Madagascar.

$\mathrm{Li}$ Yuan,Stephen Powell. MOOCs and Open Education:Implications for Higher Education.http://publications.cetis.ac.uk/wp-content/uploads/2013/03/MOOCs- and-OpenEducation.pdf, 2013.05.29.

Fengfeng Ke,Christopher Hoadley. Evaluating online learning communities. Educational Technology Research and Development .2009 (4):27-59.

Mingzhu Qiu,Jim Hewitt,Clare Brett. Online class size, note reading, note writing and collaborative discourse.International Journal of Computer-Supported Collaborative Learning .2012 (3):255-270.

Deng Hui "Faced MOOC, a Chinese university to be bold, second innovation" - Interview with Professor edX main Xianante • Agarwal Guangming Daily, 2013-10-11.

Liuzeng Hui late Chinese MOOC when a storm struck MOOC China Distance Education, 2013, (14): 12-15.

Qiong .MOOCs integration with the existing teaching Universities, for example Chinese education information • Higher Vocational Education, 2013 (6): 25-36.

Inge deWaard, Sean Abajian, Michael S.Gallagher, Rebecca Hogue, Nilgun Keskin, Apostolos Koutropoulos, Osvaldo C. Rodriguez. Using mLearning and MOOCs to Understand 